\title{
Die Nullstufe der Bühne: Spielfläche und Schranken als historische Aufführungsdispositive im spätmittelalterlichen Theater
}

\author{
Hans Rudolf Velten
}

Online publiziert: 15 . Oktober 2020

(C) Der/die Autor(en) 2020

Zusammenfassung Der Beitrag wendet sich gegen die vorherrschende Auffassung, die Simultanbühne sei die >Grundform der Raumordnung < und zentraler Repräsentationstypus des mittelalterlichen Schauspiels und lege paradigmatisch dessen ästhetische und theaterhistorische Alterität in der Differenz zur neuzeitlichen Sukzessionsbühne offen. Gegen diese verkürzende Sichtweise wird die Vielheit von räumlichen Aufführungssituationen im geistlichen und weltlichen Schauspiel dokumentiert und insbesondere die Bedeutung der kreisförmigen, durch Schranken abgegrenzten Spielfläche anhand der Tiroler Neidharttradition und der Rosengartenspiele herausgearbeitet. Abschließend werden in zwölf Punkten Funktionalität und Medialität von Schranken als Aufführungsdispositiv skizziert.

Schlüsselwörter Simultanbühne $\cdot$ Sukzessionsbühne $\cdot$ Spielfläche $\cdot$ Schranken · Neidhartszenar · Prozessionalität · Turnierspiel · mittelalterliches Schauspiel 


\title{
Staging Degree Zero: Rink and Barriers as Historical Performance Dispositifs in Late Medieval Theatre
}

\begin{abstract}
The article opposes the prevailing view that the simultaneous central stage was the >predominant form of spatial setting < and the main representational type of medieval drama, paradigmatically revealing its aesthetic and historical alterity in difference to the modern succession stage. In contrast to this generic view, the paper documents the high variety of different spatial performance situations in the sacred and secular theatre. In particular, it elaborates the significance of the circular playing area (rink) delimited by barriers, focusing the Tyrolean tradition of Neidhart plays and the Rosengarten plays. Finally, the functionality and mediality of barriers as important performance devices are being outlined in twelve points.
\end{abstract}

Keywords Simultaneous Staging · Rink · Barriers · Theatre History · Neidhart Plays · Late Medieval Drama · Playing Area · Theatrical Performance · Spatial Performance

\section{Rahmenkonzepte: zwischen Simultan- und Sukzessionsbühne}

Spätestens seit Hans Ulrich Gumbrechts Thesen zu den Übergängen vom mittelalterlichen zum neuzeitlichen Theaterrahmen gilt die Simultanbühne als das bestimmende Dispositiv für das mittelalterliche Theater. ${ }^{1}$ Sie wird, so die vorherrschende Auffassung, im Lauf des späten 15. und 16. Jahrhunderts abgelöst von der humanistischen Sukzessionsbühne, sodass mittelalterlicher und neuzeitlicher theatraler Raum auf die Gegenbegriffe > simultan< und >sukzessiv< verkürzt werden. ${ }^{2}$ Dieses Narrativ ist theatergeschichtlich deshalb so bedeutsam, weil mit dem Raum, den diese unterschiedlichen Bühnenformen organisieren, auch eine bestimmte theatrale Räumlichkeit, ein historisches Raumverständnis und sogar ein Verständnis von Spiel/ Theater überhaupt einhergeht. ${ }^{3}$ Daher sind historische Bühnenformen als äußerliche

\footnotetext{
1 Vgl. Gumbrecht, Hans Ulrich: »Für eine Erfindung des mittelalterlichen Theaters aus der Perspektive der Frühen Neuzeit«. In: Johannes Janota u. a. (Hg.): Festschrift Walter Haug und Burghart Wachinger. Tübingen 1992, Bd. 2, S. 827-848, hier S. 840.

2 So etwa theaterhistorisch Kindermann, Heinz: Das Theaterpublikum des Mittelalters. Salzburg 1980, S. 19u. 25, literaturwissenschaftlich Lösch, Matthias: »Bühne, Bühnenform«. In: Klaus Weimar (Hg.): RLW 1 (1997), S. 269-274, hier S. 270 und germanistisch-mediävistisch Schulze, Ursula: Geistliche Spiele im Mittelalter und in der Frühen Neuzeit: von der liturgischen Feier zum Schauspiel. Eine Einführung. Berlin 2012, S. 37. Ein Beispiel früher Theatergeschichtsschreibung nach Bühnenformen ist Tydeman, William: English Medieval Theatre 1400-1500. London/Boston 1986.

3 Vgl. Kreuder, Friedemann: »Hören, Gehen und Sehen. Schrift, Raum und Bild. Zur verunörtlichenden Medialität der Simultanbühne des Geistlichen Spiels«. In: Matthias Däumer/Annette Gerok-Reiter/ Friedemann Kreuder (Hg.): Unorte. Spielarten einer verlorenen Verortung. Kulturwissenschaftliche Perspektiven. Bielefeld 2014, S. 159-178, hier S. 174, im Anschluss an Erika Fischer-Lichtes Ästhetik des Performativen. Frankfurt a. M. 2004, S. 187: »Räumlichkeit einer Aufführung entsteht im und durch den performativen Raum, sie wird unter den von ihm gesetzten Bedingungen wahrgenommen.«.
} 
räumliche Strukturen entscheidende Dispositive für mediale Konfigurationen von Theatralität in unterschiedlichen Kulturen und Epochen. ${ }^{4}$

Der Simultanbühne wird ein mittelalterliches Aufführungsverständnis zugeschrieben, welches heilsgeschichtliche mit nicht-liturgischen symbolischen Inhalten auf einer Ebene der Gleichzeitigkeit verknüpft und die Ordnung und Bewegung von Darstellern und Zuschauern nach rituellen Vorgaben organisiert. Idealtypisch wird bei der Bühnenform von einem zentralen flächigen Gerüst (bis zu 100 Metern Länge) ausgegangen, auf dem sich an den Rändern aus Holz gebaute Unterstände (sogenannte loca oder mansiones) befinden, in die sich die einzelnen Darsteller und Darstellergruppen zurückziehen können, wenn sie keine Sprecheinsätze haben oder nicht in Bewegung sind. ${ }^{5}$ Hansjürgen Linke beschreibt diese Besonderheit wie folgt: »Solange sie dort saßen, waren sie nicht >da<. Erst Aufstehen bedeutete Auftreten, Hinsetzen entsprechend Abgehen. ${ }^{6}$ An dieser Aussage lässt sich erkennen, wie sehr die Beschreibung mittelalterlicher Bühnenverhältnisse selbst bei einem Spezialisten wie Linke von den Bühnendispositiven der Neuzeit (Vergleich mit Auftreten und Abgehen) konditioniert ist. Tatsächlich tritt im mittelalterlichen Spiel niemand auf und $\mathrm{ab}$, sondern alle sind gleichzeitig und von Beginn an präsent. Glenn Ehrstine hat gezeigt, dass die körperliche Präsenz der Darsteller auch dann wahrgenommen wurde, wenn die Darsteller sprachlich nicht in Aktion waren. ${ }^{7}$

Weitere Punkte für die Indienstnahme der Simultanbühne als >Grundform der Raumordnung< (Roselt) des mittelalterlichen Schauspiels sind ihre Polarität als topologische Grundlage und ihre Ermöglichung prozessionaler Bewegung. Simultanbühnen waren erstens nach dem Prinzip der christlichen Eschatologie aufgebaut: so etwa die Passionsspiele von Alsfeld, Villingen und Luzern, die den Bühnenraum innerhalb der sich an den Schmalseiten gegenüber liegenden Pole Himmel und Hölle aufspannten, zwischen denen sich die irdische Welt mit ihren zahlreichen Bühnenständen ausbreitete. ${ }^{8}$ So war es möglich, Heilsgeschichte und Gegenwart in ein Bezugskontinuum zu bringen, Immanenz und Transzendenz in einem einzigen Raum zu verknüpfen, was für die Paränese des Geistlichen Spiels fundamental war. Dieser Aspekt ist unter dem Stichwort >Präsenz< in den letzten Jahrzehnten gut erforscht worden. ${ }^{9}$ Zweitens folgten die Zuschauer den prozessionalen Bewegungen und den bildhaften Gesten (figurae) der Darstellergruppen auf der Bühne nicht nur

\footnotetext{
${ }^{4}$ Vgl. Roselt, Jens: »Raum«. In: Erika Fischer-Lichte/Doris Kolesch/Matthias Warstat (Hg.): Metzler Lexikon Theatertheorie. Stuttgart/Weimar 2005, S. 260-267, hier S. 260: »Durch den R[aum] wird die Art und Weise organisiert, wie Akteure und Zuschauer zueinander ins Verhältnis gesetzt werden. Seine Anordnung weist den Beteiligten ihre Funktionen zu, grenzt ihren Wirkungskreis ein und hat damit wesentlichen Einfluss auf die [...] Medialität von Aufführungen«.

5 Vgl. Kotte, Andreas: Theatergeschichte. Eine Einführung. Köln/Weimar/Wien 2013, S. 131.

${ }^{6}$ Linke, Hansjürgen: »Vom Sakrament bis zum Exkrement. Ein Überblick über Drama und Theater des deutschen Mittelalters«. In: Günter Holtus (Hg.): Theaterwesen und dramatische Literatur. Beiträge zur Geschichte des Theaters. Tübingen 1987, S. 127-164, hier S. 151.

${ }^{7}$ Vgl. Ehrstine, Glenn: »Das figurierte Gedächtnis: Figura, Memoria und Simultanbühne des deutschen Mittelalters«. In: Ursula Peters (Hg.): Text und Kultur. Mittelalterliche Literatur, 1150-1450. DFG-Symposion 2000. Stuttgart 2001, S. 414-437.
}

8 Vgl. Roselt (s. Anm. 4), S. 261.

9 Vgl. dazu Erstine (s. Anm. 7). 
mit den Augen, sondern teils auch körperlich, indem sie sich selbst auch um die Bühne herum bewegten. Wie bei Prozessionen oder Aufführungen auf Wagenbühnen (in Lübeck oder in England) sind somit Bewegung und körperliche Aktivität der Zuschauer Teil der Aufführung, sie umschließen die Bühne und vollziehen die Bewegungen der Akteure nach. Theologisch und paränetisch betrachtet schuf die Simultanbühne somit »einen geistigen Spannungs- und geistlichen Entscheidungsraum [...], in dem sich jede Handlung zugleich unter dem Auge Gottes wie des auf seine Gelegenheit lauernden Versuchers vollzog. $\ll{ }^{10}$ Dieser Spannungs- und Entscheidungsraum war grundlegende Voraussetzung für die Heilserfahrung der Zuschauer und die Wirksamkeit des Sündenerlasses.

Diese Merkmale der Simultanbühne und ihre offenkundige performative und symbolische Funktion für die geistlichen Schauspiele des 15. und 16. Jahrhunderts erschienen typologisch dermaßen schlüssig, dass die Bühnenform in der Forschung zum pauschalen Repräsentationstypus und Rahmenkonzept von mittelalterlichen Theateraufführungen werden konnte. Mit den Stichworten Simultaneität, Präsenz, Polarität und Prozessionalität, die auch das Verhältnis von Darstellern und Zuschauern steuern und letztere dicht in das Spielgeschehen einbinden, wird die Alterität einer > simultanen<, den heilsgeschichtlichen Inhalten und Wahrnehmungsformen angepassten Aufführungsform gegenüber der neuzeitlichen >sukzessiven< und auf Inszenierung, Repräsentation und Illusion ausgerichteten dramatischen Disposition bis heute begründet. ${ }^{11}$

Ich möchte im Folgenden argumentieren, dass dieses Narrativ von der Herrschaft der Simultanbühne als Rahmenkonzept die pluralistische Spielwirklichkeit des mittelalterlichen Schauspiels unzulässig vereinfacht und verzerrt. Nicht nur ist dieses Narrativ aufführungshistorisch zu differenzieren, sondern es erscheint auch nötig, die Rolle der Simultanbühne als leitendes Dispositiv, welches das mittelalterliche Theater auf die Hermeneutik des geistlichen Spiels verpflichtet, zu hinterfragen. Mit der Verwendung der dichotomischen Begriffe Simultaneität und Sukzessivität tradieren sich zudem zwei weitere Positionen, die zu revidieren sind: Die erste betrifft das Postulat des Undramatischen des auf der Simultanbühne aufgeführten Schauspiels gegenüber der mit dem Humanismus wiederentdeckten aristotelischen Dramenstruktur und damit unweigerlich ein »noch nicht«, eine Vorläufigkeit bezüglich der Entstehung des neuzeitlichen Theaters, ${ }^{12}$ die zweite geht - auch in der Diskussion um Bühnenformen - immer noch von einem Primat des Zeigens und Verkörperns an Stelle einer >als-ob<-Darstellung und -Repräsentation aus, was den nach dem performative turn möglichen Zugängen nicht mehr entspricht.

\footnotetext{
10 Linke (s. Anm. 6), S. 150.

11 Vgl. Kindermann, Heinz: Bühne und Zuschauerraum. Ihre Zueinanderordnung seit der Antike. Wien 1963, S. 16: »Hier umgeben die Zuschauer nicht nur den äußeren Rand des Platzes, sondern sie ziehen mit alle den heiligen oder teuflischen Gestalten von Standort zu Standort; sie sind, jeder einzelne, persönlich in Aktion, sie schreiten mit, sie leiden und sterben mit. [....] Bühnen- und Zuschauerraum sind hier eins«.

12 So etwa Michael, Wolfgang F.: Frühformen der deutschen Bühne. Berlin 1963, S. 9, und Toepfer, Regina: »Frühneuzeitliche Wende auf der Frankfurter Bühne? Das Frankfurter Passionsspiel und Paul Rebhuns Susanna zwischen Theater und Kult«. In: Robert Seidel (Hg.): Frankfurt im Schnittpunkt der Diskurse: Strategien und Institutionen literarischer Kommunikation im späten Mittelalter und in der frühen Neuzeit. Frankfurt a. M. 2010, S. 137-161, hier S. 150.
} 


\section{Vielfalt mittelalterlicher Aufführungsformen}

Blicken wir auf die Aufführungsformen der geistlichen Spiele in den deutschsprachigen Ländern und in Europa zwischen dem 13. und dem 16. Jahrhundert, müssen wir feststellen, dass die zentrale Podiumsbühne nur eine von vielen Spielorten ist. Bei den in Kirchen stattfindenden Oster- und Weihnachtsspielen der frühen Zeit gab es kaum Platz für eine zentrale Bühne, es wurde am Altar (Grab Jesu, Krippe) und in der gesamten Kirche gespielt. ${ }^{13}$ In der Braunschweiger St. Blasius-Kirche wurden zusätzlich die Treppenstufen in das Spielfeld einbezogen. In einigen Fällen, so etwa im Tegernseer Antichristspiel, gibt es Hinweise auf hölzerne mansiones (eines mit einem Thron), die für die geistlichen Spiele errichtet wurden. ${ }^{14}$ Dies waren aber keine Bühnen, sondern dem Kirchenraum und dem Spielplan angepasste, unterschiedlich hohe Podien, die als Spiel- und Aufenthaltsorte für die Darsteller dienten. Schon Young hatte höhere $»$ Schaupodien « bei Marienspielen vermutet, ${ }^{15}$ und in der Beschreibung eines Marienspiels in Avignon 1372 durch Philippe de Mézière ist die Rede von hohen Gerüsten im Kirchenraum, auf welchen gesprochen wurde. Die Kirchenraumspiele hatten auch stärker prozessionalen Charakter, wie etwa das Magierspiel in Fleury, das ohne festen Spielort auskommt und die gesamte Kirche zum Spielraum macht, wobei die Gemeinde als Zuschauer direkt in das Spiel mit einbezogen wurde (interrogent astantes).$^{16}$ Für die englischen Mysterienspiele am Beispiel des Ludus Conventriae hatte Weimann festgestellt, dass die dort aufgebauten Gerüste nicht mit der Bühne identisch waren: »Die Spielfläche ist größer als die Summe aller Bühnengerüste, aller scaffolds oder stages. $\ll^{17}$ Bei den (lateinischen) Feiern und den Spielen im Kirchenraum handelte es sich um quasidramatische liturgische Zeremonien, für die im eigenen inhaltlichen Kontext das Zurücklegen einer Wegstrecke und die Ankunft an einem Ziel bestimmend waren: Visitatio sepulchri, officium pastorum, officium stellae, descensus, Jüngerlauf. Kirchner sieht in der prozessionalen Bewegung dieser Geschehensabläufe sogar den originären theatralen Raum des mittelalterlichen geistlichen Spiels. ${ }^{18}$ In jedem Fall lässt sich sagen, dass für die Osterfeiern und die frühen Spiele innerhalb von Kirchen eine andere Körperund Raumorganisation gilt. ${ }^{19}$

Die ersten Zeugnisse eines zentralen Podiums im deutschsprachigen Raum finden sich erst später, wenn die geistlichen Spiele vor der Kirche oder auf Marktplätzen

\footnotetext{
13 Wolfgang F. Michael betont in seinen Studien zur frühen Bühne mehrfach, dass es in den Kirchenraumspielen kein zentrales Bühnenpodium gab. Vgl. Michael (s. Anm. 12) u. ders.: »Zur Entstehung der Podiumsbühne«. In: Rolf Badenhausen/Harald Zielske (Hg.): Bühnenformen - Bühnenräume - Bühnendekorationen. Beiträge zur Entwicklung des Spielorts. Herbert A. Frenzel zum 65. Geburtstag von Freunden und wissenschaftlichen Mitstreitern. Berlin 1974, S. 9-18.

14 Vgl. Michael (s. Anm. 13), S. 10.

15 Vgl. Young, Karl: The Drama of the Medieval Church. 2 vol. Oxford 1933, S. 225-245.

16 Vgl. Michael (s. Anm. 12), S. 20.

17 Weimann, Robert: Shakespeare und die Tradition des Volkstheaters. Soziologie, Dramaturgie, Gestaltung. Berlin 1967, S. 127.

18 Vgl. Kirchner, Thomas: Raumerfahrung im geistlichen Spiel des Mittelalters. Frankfurt a.M./Bern/New York 1985, S. 26.

19 Vgl. Schulze (s. Anm. 2), S. 221.
} 
aufgeführt wurden. So gibt es ab 1430 in Tirol Zeugnisse über Gesamtbühnen (in Halle wird von einem gerüst zuem Spil gesprochen), die vollgültig als Simultanbühnen bezeichnet werden können. Erst im 16. Jahrhundert scheint sich in Tirol die Gesamtbühne durchgesetzt zu haben (in Bozen sogar innerhalb der Kirche). ${ }^{20}$

Selbst die berühmteste Simultanbühne der Theatergeschichte nach dem Bühnenplan des Luzerner Weinmarkts von Renward Cysat 1583 ist eigentlich eine SimultanRaumbühne. Der Weinmarkt (damals Fischmarkt) ist ein Platz von ca. $60 \mathrm{~m}$ Länge und ca. $20 \mathrm{~m}$ Breite, welcher im Osten durch das Haus >Zur Sonne< und im westlichen Abschnitt mit dem >Schönen Brunnen< begrenzt ist. Zwei Drittel des Platzes dazwischen (die platea) wurde für die Simultan-Raumbühne der Luzerner Passionsspieltradition genutzt. Dies ist also eher ein umgrenzter Spielraum und kein Bühnengerüst wie in anderen Städten, wo man Simultanbühnen auf Gerüsten von einem halben bis einem Meter Höhe errichtete. Um die platea herum waren auf hölzernen Plattformen die höff, huss oder ort der einzelnen Figuren und Figurengruppen errichtet. Bis zu viertausend Zuschauer fanden hier Platz - teils auf einer großen Zuschauertribüne im westlichen Drittel des Platzes, teils auf den Dächern der höff und hus; die Fenster der Bürgerhäuser wurden als >Logen< genutzt, in welchen auch zahlreiche Zuschauer unterkommen konnten. ${ }^{21}$ Da die Zuschauer fest an ihren Plätzen standen oder saßen, kann von einer prozessionalen Begleitung der Darsteller nur für wenige von ihnen die Rede sein. Diese späten Passionsspielaufführungen entsprachen somit nicht mehr der prozessionalen Rezeptionsform. Diese war noch von 1453 bis 1500 in Luzern ausgeübt worden. Man spielte in dieser Zeit (und vielleicht auch früher) nicht auf dem Weinmarkt, sondern auf dem kleineren Kapellplatz. Für die frühen Spiele von 1453 und 1470 kommt »nur eine prozessionale Aufführungsform in Frage, bei der Darsteller wie Zuschauer von Schauplatz zu Schauplatz zogen, wobei gewisse Szenen - beispielsweise die einzelnen Stationen des Kreuzwegs - auf der gemein bürge, auf dem freien Platz in der Mitte, gespielt wurden «, so Greco-Kaufmann. ${ }^{22}$ Dort ließ die räumliche Situation den Aufbau von Tribünen nicht zu. Die Zuschauer versammelten sich vor den jeweiligen Höfen oder Schauplätzen, um das Geschehen aus der Nähe verfolgen zu können. Sie mussten sich dabei für jede Szene neu gruppieren und den Darstellern auf dem Platz processionaliter nachziehen. Dadurch wurde beim Passionsspiel eine Analogie zum Kreuzweg Christi erreicht, im Sinne eines performativen, körperlich-symbolischen Nachvollzugs des Leidenswegs Jesu. So konnte in der Erinnerung der Kapellplatz als heiliger Ort symbolisch besetzt werden. ${ }^{23}$ Auch bei anderen Prozessionsspielen wie in Künzelsau, Bozen, Freiburg und Zerbst wurde nicht oder nur teilweise auf Bühnen gespielt, sondern an den einzelnen Stationen der Prozession, an welchen die Spielgruppen figurae als lebende Bilder, teils in pantomimischer Form (Zerbst),

\footnotetext{
20 Vgl. Michael (s. Anm. 13), S. 13.

21 Vgl. die genauen Beschreibungen bei Greco-Kaufmann, Heidy: ‘Zuo der Eere Gottes, vfferbuwung dess mentschen vnd der statt Lucern lob . Theater und szenische Vorgänge in der Stadt Luzern im Spätmittelalter und in der Frühen Neuzeit. Bd. 1. Zürich 2009, S. 433-483.

22 Ebd., S. 173.

23 Vgl. Kotte (s. Anm. 5), S. 136.
} 
inszenierten. ${ }^{24}$ Simultanbühnen waren bei Prozessionsspielen die Ausnahme - sie kamen etwa in Bozen und Freiburg im 16. Jahrhundert zur Anwendung. Überhaupt kann mit Wolfgang F. Michael gesagt werden: Erst in der zweiten Hälfte des 16. Jahrhunderts $»$ scheint die Bühne auf einem großen Podium die vorherrschende Form geworden zu sein ${ }^{25}$

Beim weltlichen Schauspiel ist die Situation noch differenzierter. Die beiden größten Fastnachtspieltraditionen in Nürnberg (Einkehrspiele) und Lübeck (Wagenspiele) arbeiten ohne eigentliche >feste< Bühne: Bei den Einkehrspielen betrat die Spielgruppe das Wirtshaus oder ein Privathaus und spielte dort in einem größeren Raum vor Publikum. Es wurde spontan durch raumschaffende Maßnahmen ein runder Spielkreis, in den Quellen ring oder circulus genannt, für das Spiel der Darsteller geschaffen, während die Zuschauer um diesen Spielkreis herum standen. ${ }^{26}$ An den Ansprachen des Präcursors und auch der Figuren ist die enge Kommunikation mit dem Publikum zu erkennen. Beginn und Ende wurde durch Musik (Pfeifer oder Lautenspieler) und Tanz gerahmt. ${ }^{27}$ Bei den Lübecker Wagenspielen, die ab 1430 durch die Zirkelbruderschaft aufgeführt wurden, handelte es sich um fahrbare Schaubühnen, die offenbar aus Flandern eingeführt worden waren. Die Spielgruppen spielten sowohl auf verschiedenen Plätzen der Stadt als auch vor der Wagenbühne direkt vor den Zuschauern. Den Spielwagen folgten auch hier Straßentänze mit musikalischer Begleitung als verbindende Elemente von Spiel und Karnevalsritualen. ${ }^{28}$ Die Fastnachtspiele waren somit in ihrer Aufführung stark an die dispersive räumliche Organisation des Karnevals gebunden, was eine deutliche Differenz zu den auf einen bestimmten Ort fokussierten städtischen Großereignissen der geistlichen Spiele darstellte.

Auch für die im süddeutschen und im Alpenraum verbreiteten Neidhartspiele ist kein Podium nachweisbar. Das Große Neidhartspiel in Tirol war als Freilichtaufführung mit verschiedenen stant für die Spieler konzipiert, während das Tiroler (Mittlere) Neidhartspiel offenbar 1511 auf einem turnierartig umschrankten Platz mit dramaturgisch aufeinander ausgerichteten ort inszenierte. ${ }^{29}$

Fastnächtliche Rathausspiele wurden nach Eckehard Simon in den Tanzsälen der Rathäuser, also in größeren Innenräumen aufgeführt und offiziell gefördert, wobei die Quellen nichts über die Aufführungsform berichten. ${ }^{30}$ Fastnächtliche Marktspiele können entweder auf Gerüsten bzw. Holzbühnen oder auch - wie die Neidhartspiele - mit Schranken aufgeführt werden (dazu mehr weiter unten). Für die meisten

\footnotetext{
24 Vgl. Michael (s. Anm. 12), S. 53-55; Ehrstine (s. Anm. 7), S. 420.

25 Michael (s. Anm. 13), S. 17.

26 Im bereits Anfang des 14. Jahrhunderts entstandenen St. Pauler Neidhartspiel etwa heißt es: Horent frawen vnde man / Ez kument her vf disen plan / Jetzo an diser vart / Ain herczogin vnd her Nithart (Margetts, John [Hg.]: Neidhartspiele. Graz 1982, S. 11, V. 2-5). plan und vart weisen auf einen offenen, unspezifischen Bühnenraum hin, der sogar auch eine Aufführung im Freien vermuten lassen könnte. Simon hält das Spiel für ein »altes fastnächtliches Einkehrspiel« (Simon, Eckehard: Die Anfänge des weltlichen deutschen Schauspiels 1370-1530. Untersuchung und Dokumentation. Tübingen 2003, S. 350).

27 Vgl. ebd., S. 177.

28 Vgl. ebd., S. 236-241.

29 Vgl. ebd., S. 356-357.

30 Vgl. ebd., S. 354-355.
} 
Aufführungen dieser längeren Spiele sind Bühnen oder Gerüste bezeugt, allerdings erst in der zweiten Hälfte des 15. und im 16. Jahrhundert.

Hinzu kommt eine ganze Reihe weiterer Aufführungsformen von fastnächtlichen Spielen und performances, die ohne jegliche Bühne auskommen, z. B. Umzugsspiele, welche ab 1484 in den Schweizer Städten belegt sind, als zehn Züricher Ratsherren als die Zehn Lebensalter verkleidet durch die Stadt zogen. ${ }^{31}$ Fastnächtliche, häufig spöttische und satirische Umzüge sind aus Bern (1521 und 1523 durch Niklaus Manuel) überliefert, aber auch in Stralsund und in verschiedenen preußischen Städten gab es Spottumzüge durch die Stadt, häufig nach dem Brauch des Pflugziehens modelliert. ${ }^{32}$ Auch textlose Fastnachtspiele benötigten kein Bühnengerüst: Jagden auf den Wilden Mann, Moriskentänze, Schwerttexte, Schiff- und Pflugziehen (teilweise auch mit aufgesagten Sprüchen), Läufe und Umzüge, kurz all das, was Simon zum »Straßentheater « zählt. $^{33}$

Überraschend ist schließlich, dass auch das frühe Humanistendrama ohne Bühne auskam, da es sich dabei um Aufführungen von Lateinschülern zum Zweck der darstellenden lateinischen Rede handelte (eine Bühne ist einzig von Locher in seinem Türkenschauspiel von 1497 überliefert). ${ }^{34}$ Das Augsburger Schultheater von Sixt Birck wurde im Schulhof aufgeführt - auch ohne Bühne, wohingegen Sebastian Wild in Straßburg ein Gerüst und sogar Vorhänge (nach der Terenztradition) benutzte. ${ }^{35}$ Auch die ersten Fastnachtspiele von Hans Sachs sind für eine »sehr einfache, ortlose, podiumslose Bühne in kleinem Raume geschrieben «. Um die Jahrhundertmitte verwendet Sachs für seine comedis und tragedis dann ein Bühnenpodium und einen »immer komplizierteren Bühnenapparat «. ${ }^{36}$ Erst mit diesen Dramen und den komplexeren Fastnachtspielen aus dieser Zeit, von denen vermutlich viele auch in der Marthakirche aufgeführt wurden, verbreitete sich schließlich die Podiumsbühne in Deutschland. ${ }^{37}$

\section{Das Verhältnis von Darstellern und Zuschauern}

Mit der Mannigfaltigkeit der Aufführungsformen bei geistlichen und weltlichen Spielen von 1300-1600 bleiben auch vereinheitlichende Postulate zum Verhältnis zwischen Darstellern und Zuschauern und zur Wahrnehmungsästhetik des Geistlichen Spiels auf der Strecke. Kindermann etwa hatte einen Wandel der Bühnensituation in der ersten Hälfte des 16. Jahrhunderts angenommen, indem das humanistische Theater die Kreisform verlassen hätte. Der Übergang von der Simultan- zur Sukzessionsbühne habe ein »Moment der Distanz« zwischen Spiel- und Zuschauerwelt eingeführt; die Zuschauer seien nicht mehr »Ergriffene« wie im geistlichen Spiel

\footnotetext{
31 Vgl. ebd., S. 358.

32 Vgl. ebd.

33 Ebd., S. 186-190.

34 Vgl. Michael (s. Anm. 13), S. 14.

35 Vgl. ebd.

36 Ebd., S. 17u. 17 f.

37 Vgl. ebd., S. 18.
} 
des Mittelalters, sondern, abgetrennt von der Spielwelt, »Betrachtende «. ${ }^{38}$ Dieser eingängig scheinende Wandel in der Zuschauerhaltung, der symptomatisch den Beginn des Illusionstheaters anzeigen soll, ist nichts weiter als eine simplifizierende Hypostasierung. Weder sind die Zuschauer der mittelalterlichen Schauspiele immer ergriffen und von der Spielwelt ungeschieden, weder sind sie immer kreisförmig gruppiert, noch besitzt das Humanistendrama, wie oben erwähnt, die ihm hier zugeschriebenen neuartigen Qualitäten. Ist es schon schwierig genug, bei einer frühen Osterfeier wie der Regularis Concordia (Winchester 973), die in den Didaskalien eindeutige >als-ob<-Anweisung zu interpretieren und sie hier von jeglicher Form der spielerischen Inszenierung auszuschließen, so oszilliert auch später das Geistliche Spiel noch zwischen Realpräsenz und Repräsentation, ${ }^{39}$ ganz zu schweigen von den weltlichen Spielen, die dieser heilsgeschichtlichen Wirkungsfunktion (Sündenerlass) gar nicht dienstbar waren.

Der Mannigfaltigkeit der Bühnenform im mittelalterlichen Schauspiel entspricht auch eine größer als bisher angenommene Varianz des Zuschauerverhaltens. So stehen die silete-Rufe der Engel und die Ermahnungen der Spielleiter und Proklamatoren an das Publikum, zu schweigen, in diametralem Gegensatz zur fastnächtlichen Ausgelassenheit und lautstarken Teilhabe des Publikums von Fastnachts- und Neidhartspielen. Im Frankfurter Passionsspiel etwa müssen die Zuschauer eine passive Rezeptionshaltung einnehmen: Sie dürfen das Spiel nicht durch Zwischenrufe unterbrechen und sollen dem Geschehen aufmerksam folgen. ${ }^{40}$ Der Kirchenvater Augustinus, der als Proklamator das Spiel eröffnet, zieht bereits in seiner ersten Ansprache eine Grenze zwischen Darstellern und Zuschauern: Ir hirschafft, stellet uwern schalle, / dis wart vernemet uber alle (V. $1 \mathrm{f}$.). ${ }^{41}$ Die paränetisch-didaktische Ausrichtung des Spiels macht so die mit der Simultanbühne geschaffene Durchlässigkeit zwischen Darstellern und Publikum zunichte: Letzteres ist aufgefordert, über auditive und visuelle Teilhabe das Spielgeschehen zu verinnerlichen, aber durchaus in Distanz zu ihm. Genau dies brachte Luther auch zu seinem kritischen Urteil, die Geistlichen Spiele veranlassten die Zuschauer zu einer passiven Rezeptionshaltung, um göttliche Präsenz zu erfahren..$^{42}$

Zwar ist man übereingekommen, das Verhältnis von Darstellern und Rollen im geistlichen Spiel allgemein mit dem Begriff der >stellvertretenden Verkörperung < zu kennzeichnen, d.h. eine zeichenhafte Präsenz der Darsteller anzunehmen, welche sowohl Redeeinsätze als auch Gestik in den figurae umfasst, doch dies führt nicht

\footnotetext{
38 Kindermann (s. Anm. 11), S. 19-23.

39 Jan-Dirk Müller hat für den beginnenden mimetischen Charakter des Spiels, das zwischen Realpräsenz und Repräsentation oszilliert, den Begriff der »Theatralen Mimesis« geprägt (ders.: »Realpräsenz und Repräsentation. Theatrale Frömmigkeit und Geistliches Spiel«. In: Hans-Joachim Ziegeler (Hg.): Ritual und Inszenierung. Geistliches und weltliches Drama des Mittelalters und der Frühen Neuzeit. Tübingen 2004, S. 113-133, hier S. 122).

40 Vgl. Toepfer (s. Anm. 12), S. 145.

41 »Frankfurter Passionsspiel«. In: Johannes Janota (Hg.): Die hessische Passionsspielgruppe. Edition im Paralleldruck. Bd. 1: Frankfurter Dirigierrolle, Frankfurter Passionsspiel. Tübingen 1996, S. 53-430, hier S. 69.

42 Vgl. Luther, Martin: WA Bd. 2: Schriften 1518/19. Weimar 1884 in seinem Sermon der Betrachtung des heyligen Leydens Christi, S. 131-142.
} 
zwangsläufig zu einer identischen Rezeptionshaltung der Zuschauer in den verschiedenen Spieltypen; vor allem in Bezug auf die Frage der Qualität und der Struktur von Bewegung der Zuschauer im Raum muss hier doch die stumme, regungslose Betrachtung von dem körperlich bewegten, performativen Nachvollzug in prozessionalen Aufführungen unterschieden werden. ${ }^{43}$ Die Bewegung und das Handeln im Raum stellen einen grundsätzlich divergenten Rezeptionstypus als die meditative Andachtsschau dar, welche mit dem Betrachten der Szenen auf der Simultanbühne verbunden war. Bei prozessional organisierten Spielen (wie dem Künzelsauer Fronleichnamsspiel) und Spielabschnitten herrscht eine Teilhabe über körperlichen und haptischen Mit- und Nachvollzug vor, die mit dem Begriff des »Handelns im Raum $\aleph^{44}$ treffend gekennzeichnet wurde, selbst wenn diese Spiele ihre figurae teilweise auch auf Simultanbühnen aufführten. ${ }^{45}$ Die Bewegungssukzession des prozessionalen Modus und seine spezifische Wahrnehmungsform nähern die Zuschauer im »Modell des Weges zur himmlischen Heimat « (Wilhelm Durandus) ${ }^{46}$ den Darstellern und ihren figurae an, da sich diese sowohl bei Ein- und Auszug, aber auch während des Spiels processionaliter bewegen. Werner Röcke hat sogar im Anschluss an Rudolf Otto von einer »Annäherung an das Heilige « als das »ganz Andere« gesprochen. ${ }^{47}$ Zumindest wird etwa die geforderte imitatio Christi oder der klagenden Frauen bei der prozessionalen Wahrnehmung auf eine andere Grundlage gestellt. Ein- und Auszüge folgen zudem einer intrikaten Choreographie, indem etwa die Darsteller in der Reihenfolge ihres Auftretens oder in der Reihenfolge ihrer Bedeutung für das Spiel vorüberschritten, sodass hier die Handlung gewissermaßen schon antizipiert wurde. ${ }^{48}$ Auch die um die Spielorte der figurae häufig kreisförmig sich bewegenden Darsteller, welche die Raumgrenze gleichzeitig konstituieren und durchbrechen (dabei kommt es zu Grenzüberschreitungen von Heiligkeit und Profanität, Immanenz und Transzendenz), können von den in Bewegung befindlichen Zuschauern viel besser nachvollzogen und rememoriert werden, als wenn sie diese Bewegungen nur meditativ betrachtet hätten. Andererseits konnten etwa die Zuschauer der Luzerner Passionsspiele auf dem Weinmarkt von ihren Häusern und von der Tribüne aus das Geschehen auf der Simultan-Raumbühne audiovisuell viel besser und aufmerksamer verfolgen als noch im 15. Jahrhundert, als die Spiele im prozessionalen Modus auf dem Kapellplatz aufgeführt wurden und die Zuschauer in einer großen Menge eingebunden waren. Man könnte hier sogar von einem histo-

\footnotetext{
43 Ehrstine (s. Anm. 7) vermeidet in seiner Untersuchung der Prozessionsspiele diese Unterscheidung und geht von einer einheitlichen figura-Rezeption als Grundhaltung bei allen geistlichen Spielen aus, unabhängig von ihrer Aufführungsform.

44 Kirchner (s. Anm. 18), S. 25.

45 So etwa in Zerbst und Freiburg, vgl. Ehrstine (s. Anm. 7), S. $430 \mathrm{f}$.

46 Vgl. dazu Kiening, Christian: »Prozessionalität der Passion«. In: Katja Gvozdeva/Hans Rudolf Velten (Hg.): Medialität der Prozession. Performanz ritueller Bewegung in Texten und Bildern der Vormoderne. Médialité de la procession. Performance du mouvement rituel en textes et en images à l'époque prémoderne. Heidelberg 2011, S. 177-198, hier S. 177.

47 Röcke, Werner: »Realpräsenz des Heiligen und karnevaleske Verkehrung. Annäherungen an das >ganz Andere in geistlichen und weltlichen Prozessionsspielen des Mittelalters«. In: Katja Gvozdeva/Hans Rudolf Velten (s. Anm. 46), S. 307-322, hier S. 309.

48 Belegstellen bei Ehrstine (s. Anm. 7), S. 436.
} 
rischen Wandel in Richtung Trennung von Darstellern und Zuschauern sprechen. ${ }^{49}$ Es macht somit einen Unterschied, ob die Zuschauer eine prozessionale oder eine statische Rezeptionshaltung einnehmen.

Prozessionen sind auch bei den weltlichen Spielen substantieller Bestandteil der Aufführung; sei es bei den fastnächtlichen Einkehrspielen, die von Ein- und Auszügen und Tanz gerahmt werden, sei es bei den Neidhartspielen, welche auch innerhalb der Spiele von Musik und Tanz bestimmte Gruppenchoreographien aufweisen, oder den beweglichen Bühnen der Wagenspiele. Der prozessionale Modus ist »das entscheidende tertium comparationis « der Fastnacht- und der Fronleichnamsspiele, auch wenn es sich dabei um gänzlich unterschiedliche Formen von Prozessionen handelte..$^{50}$ Dies gilt natürlich ebenso für alle textlosen Spiele, Läufe und Rituale, die weder gespannte Aufmerksamkeit beim Zuschauen erfordern noch mit heilsgeschichtlicher Vermittlung beauftragt sind. Dass schließlich die Rezeptionshaltung der Zuschauer in vielen weltlichen Spieltypen (bis auf die zentrale Podiumsbühne bei Marktspielen) sich grundlegend von jener der geistlichen Spiele unterscheidet, ist oft betont worden. So sind Akteure und Publikum bei Einkehrspielen (und vermutlich auch bei Wagenspielen) kaum voneinander distanziert und in enger Interaktion miteinander verbunden (Ansprachen, Zwischenrufe, Fragen und Antworten, Tanz, Lachanlass und Gelächter).

Schließlich ist es aus pragmatischen Gründen schwierig, von einer einheitlichen Rezeptionshaltung der Zuschauer allein im Geistlichen Spiel auszugehen. Das Simultane der Simultanbühne wird nämlich dann zum Phantasma, wenn nur ein Teil der Zuschauer dieser Simultaneität gegenwärtig werden kann, weil nur er das gesamte Geschehen zu überblicken vermag. Viele Zuschauer sehen bei den mittelalterlichen Aufführungen eben nicht alles, und sie hören auch nicht unbedingt alles, da sie sich in größerer Entfernung vom Spiel befinden. Auch haben sie je nach Standort unterschiedliche Perspektiven auf den Bühnenraum, der eventuell teilweise verdeckt wird.

\section{Offene Spielflächen und Schranken}

Ich möchte im Folgenden nun auf einen Aufführungstyp eingehen, der von der Forschung bisher wenig beachtet wurde, aber doch verbreiteter war als bislang gesehen. Es handelt sich dabei um die offene Spielfläche auf einem öffentlichen Platz, der wie bei einem Turnier durch Schranken begrenzt wird, welche eine (poröse) Grenze zwischen Darstellern und Zuschauern instituieren. Der umschrankte Spielraum ist einer jener Aufführungsdispositive mittelalterlichen Theaters, die vor allem für weltliche Spielformen charakteristisch, aber auch für geistliche Spiele belegt sind und sich in Modus und Wirkung von jenem der Simultanbühne mehr oder weniger stark unterscheiden.

Hinweise auf Schranken in den Aufführungsbelegen zum Geistlichen Spiel sind gering. In Bozen scheint man die Bühne umschrankt zu haben, in Luzern wurden

\footnotetext{
49 Vgl. dazu Kotte (s. Anm. 5), S. 136.
}

50 Röcke (s. Anm. 47), S. 309. 
bereits im 15. Jahrhundert Schranken bestellt (Item dass osterspyll zuo schrancken etc.), in Kitzbühel wurden den Zimmerleuten für die Schranken für das Osterspiel 20 Kreuzer gegeben und auch in Weilheim wurden Schranken im Zusammenhang mit dem Aufbau der Bühne gefertigt. In Burghausen gebrauchte man Schranken bei der Fronleichnamsprozession. ${ }^{51}$ Aus Basel berichtet Johannes Gast 1546 anlässlich des Spiels von der Bekehrung Pauli von hölzernen Schranken (cancellis), die zwischen Adel und Volk aufgebaut waren. ${ }^{52}$ Das Beispiel zeigt, dass Schranken nicht allein zur Abgrenzung des Spielfelds von den Zuschauern benutzt wurden, sondern auch zur ständischen Abgrenzung verschiedener Zuschauergruppen oder um Spaliere für Ein- und Auszüge herzustellen. Welche dieser Funktionen sie im zur hessischen Spielgruppe gehörigen Alsfelder Passionsspiel hatten, dem berühmtesten Beispiel eines Geistlichen Spiels, das offensichtlich eine umschrankte Raumbühne vorsah, ist nicht mit Bestimmtheit zu sagen. ${ }^{53}$ Dort ist von einem krey $\beta$ die Rede, der mit einem abgezirkelten runden Spielfeld identifiziert werden kann, in welches die Zuschauer nicht eintreten durften: Wer da betredden wirt in dissem kreyß / er sij Heyncz. adder Concz adder wie er hey $\beta$ / der do nit gehoret in dit spiel (V. 111-113), ${ }^{54}$ so der Proklamator zu Beginn. Er grenzt mit Hilfe dieses Zutrittsverbots somit zunächst verbal den Spiel- vom Zuschauerraum ab und fordert den Schultheiß auf, in einem symbolischen Rechtsakt die Gültigkeit der Abgrenzung der Spielfläche, die offenkundig bereits durch Schranken abgetrennt ist, mit zu markieren: her schultheys, macht ir den slagk / do sich eyn iglicher nach richten magk (V. 123 f.). ${ }^{55}$ Ein bildliches Beispiel von hölzernen, aus Weiden geflochtenen Schranken ist die berühmte Darstellung Jean Fouquets des Spiels vom Martyrium der Heiligen Apollonia, wo man die mit Blättern und Zweigen dekorierte Beschrankung im Vordergrund erkennen kann.

Wichtiger als im Geistlichen Spiel waren Schranken bei aller Art von weltlichen performances, Ein- und Auszügen, Läufen und Spielen. Das mittelhochdeutsche Wort schranke geht auf das Turnierwesen zurück und wird bei Matthias Lexer mit »gitter, zaun, schranke [...] um einen turnierplatz [...] « beschrieben, ${ }^{56}$ im Frühneu-

\footnotetext{
51 Alle Belege in Neumann, Bernd: Geistliches Schauspiel im Zeugnis der Zeit. Zur Aufführung mittelalterlicher religiöser Dramen im deutschen Sprachgebiet. 2 Bde. München/Zürich 1987: Bozen 836 und 871, Burghausen 1042, Kitzbühel 1977, Luzern 2045 und Weilheim 2838.

52 Vgl. Gast, Johannes: Tagebuch. In Auszügen behandelt von Tryphius. Übersetzt und erläutert von Karl Buxtorf-Falkeisen. Basel 1856, S. 270: [...] theatrum Magistratu ordinante ac cancellis muniente ligneis, intra quos consederant nobiles cum senatoribus; sed populus promiscuus in tribus ligneis pontibus declivibus spectatorem egit.

53 Vgl. Freise, Dorothea: Geistliche Spiele in der Stadt des ausgehenden Mittelalters. Frankfurt - Friedberg - Alsfeld. Göttingen 2002, S. 262.

54 >Alsfelder Passionsspiel<. In: Janota, Johannes (Hg.): Die hessische Passionsspielgruppe. Edition im Paralleldruck. Bd. 2: Alsfelder Passionsspiel. Edition der Melodien von Horst Brunner. Tübingen 2002, S. 233.

55 Ebd. Im Mhd. bedeutet slac / slag auch »schlagbaum, schranke, keyo, phala, phalanga, repagulum«; Bd. 2, Sp. 950 f. Vgl. dazu auch Vogelgsang, Klaus (Hg.): Die Hessische Passionsspielgruppe. Ergänzungsband 2: Kommentar zum >Alsfelder Passionsspiek und den zugehörigen kleineren Spielzeugnissen, Berlin 2007, S. 64 u. Ackermann, Christiane: »Schwellengänge in Raum und Zeit: Immersion im geistlichen Spiel«. In: LiLi 167 (2012), S. 82-103, hier S. 91 f.

56 Lexer, Matthias: Mittelhochdeutsches Handwörterbuch (1973-1976). Bd. II, Sp. 786.
} 
hochdeutschen wird >schranke< auch noch spezifischer als eine »absperrung eines raumes für kampf, turnier, spiel, rennen, um schau- und andere plätze « definiert. ${ }^{57}$ Insbesondere auf Markt- und anderen Plätzen in Städten oder auf Festangern vor den Stadtmauern wurden spätestens seit dem hohen Mittelalter Schranken verwendet, um Turniere und Wettspiele auszutragen, ihren geregelten Ablauf zu gewährleisten und die Zuschauer vor Schaden zu bewahren. Schranken hatten den Vorteil, dass sie beweglich waren und so die Spielfläche in flexibler Weise umgrenzen konnten. Sie stellen sicherlich, neben der ort- und schrankenlosen Bühne (wie im fastnächtlichen Einkehrspiel), die allein durch verbale und gestische raumschaffende Maßnahmen gebildet wird (durch den Präcursor oder einen Narren), eine der basalsten Formen der Abgrenzung zwischen Darstellern und Zuschauern bei einer performance dar. Im Moment der Aufführung eines theatralen Schauspiels, dessen Spielplan oder Bühne durch kreis- oder halbkreisförmig aufgestellte Schranken konstituiert wird, stellt sich die Frage nach den besonderen historischen Bedingungen, den Darstellungs- und Wahrnehmungsmöglichkeiten, die durch diese einfache Bühnenformen ermöglicht oder ausgeschlossen werden. ${ }^{58}$

Ich will dies im Folgenden anhand von zwei Spieltypen des weltlichen Schauspiels näher erläutern: den Neidhartspielen, insbesondere dem Tiroler (mittleren) Neidhartszenar (auch: Sterzinger Neidhartszenar) und den Aufführungen von Rosengärten.

\subsection{Das Neidhartszenar}

Linke hatte bei seiner Autopsie der in Kanzleischrift geschriebenen Papierhandschriften der beiden Sterzinger (Tiroler) Neidhartspiele im Gegensatz zur älteren Forschung (A. Dörrer) festgestellt, dass das Mittlere Tiroler Spiel und das Neidhartszenar von derselben Hand geschrieben wurden und auch textuell nahezu identisch sind (nur fünf leicht abweichende Stellen); beide Handschriften seien gegen 1511 in Bozen oder Umgebung entstanden. ${ }^{59}$ Er identifiziert das Szenar als veritable $>$ Dirigierrolle< des Mittleren Tiroler Neidhartspiels, in welches der Schreiber jeweils das erste Reimpaar jedes Sprecheinsatzes aufgezeichnet hat und eine Fülle von Regieanweisungen gibt. Das Regieheft war offenbar für eine geplante Aufführung gedacht, wie bereits die ersten Regieanweisungen zeigen:

\footnotetext{
57 Grimm, Jacob u. Wilhelm: Art. >Schranke[n]<. In: Deutsches Wörterbuch 15 (1899), Sp. 1634 (Bedeutung 3a) mit zahlreichen Textbeispielen, die die breite Verwendung des Begriffs im Singular und Plural belegen.

58 Roselt (s. Anm. 4) zum historischen Raum im Theater, S. 267. Roselt stützt sich dabei auf die Raumanalyse von Martina Löw, welche zwischen >spacing < und >Syntheseleistung < unterscheidet. Spacing ist die lokale Platzierung von Menschen und sozialen Gütern im Raum: Errichten, Bauen, Positionieren. Doch erst über die Wahrnehmungs-, Vorstellungs- und Erinnerungsprozesse werden Güter und Menschen zu Räumen zusammengefasst (Syntheseleistung). Raum entsteht somit erst als »Koproduktionen von Akteuren und Zuschauern an einem Ort« (ebd.).

59 Linke, Hansjürgen: »Das Tiroler (Mittlere) Neidhartspiel und seine Dirigierrolle«. In: Archiv für das Studium der neueren Sprachen und Literaturen 222 (1985), S. 1-21, hier S. 9-14.
} 
Neydthardt

Ordnunng des Neydt=

hardt Spyls etc

Wann mann am Erstn in der Ordnung

hynaus zun Schrannckn geet, So

sullen die pfeyffer voran, vnd

Etwer mit Jn geen, das Volck aus

dem wege zeweysn, ainen gerawm

zemachen,

Darnach sullen der Precursor zevördrist

vnd zwen Ritter vor der Herzogin

geen, vnd Sy, der Hofmaister

darauffüern [...].

Vnd wann Sy alle zu der Schrannckn

koment, So sullen Sy, ettliche Schryt

daruor, in der Ordnung stylle stehen,

Vnd precursor dieweyl allaine

mytten in die Schrannckn geen, vnd

also sprechen,

Hört zu Jer Frawenn vnd Jer Man,

Edl vnd vnedl auf disem plaan

Jer werdet seltzam Abentewr sehen,

das tu Jch Ew fürwar veryehen [...] (V. 1-13, 18-27)

Das Spiel findet somit auf einem von Schranken umgrenzten, kreisförmigen Platz (später wird von einem rynng die Rede sein, V. 90) im Freien (plaan) statt, zu welchem die Darsteller durch die Menge der Zuschauer sich hinbewegen. Dabei sollen vor den pfeyffern Personen gehen, die Platz für den Einzug der Darsteller machen sollen (das Volck aus dem wege [...]weysn, ainen gerawm zemachen, V. 6-8). Linke resümiert: »Man spielte also auf einem außerhalb des Ortes gelegenen Anger, der mit Holzbarrieren eingezäunt war; hinter diesen umdrängten ihn rings die $\mathrm{Zu}$ schauer, denn ein oder mehrere Spielordner [...] sind nötig, um das volck aus dem wege zeweysn $\ll{ }^{60}$ Ordner gab es auch bei den repräsentativen Schauturnieren und -stechen des Stadtadels, wenn an den Enden der eingeschrankten Bahnen Helfer

\footnotetext{
60 Ebd., S. 17. Simon vermutet im Gegensatz dazu, dass das Spiel auf dem Markt in Bozen (Musterplatz) aufgeführt worden sei; dies könnte möglich sein, jedoch spricht das hynaus zunn Schrannckhen eher für eine Freilichtaufführung vor der Stadt. Dies ist auch für die Aufführung des Großen Tiroler Neidhartspiels wahrscheinlich, da dort von verschiedenen stant für die Spieler die Rede ist. Vgl. Margetts (s. Anm. 26), S. 285, der eine Freilichtaufführung im Frühjahr annimmt, wie auch Simon (s. Anm. 26), S. 355-357, der eher für ein Marktspiel plädiert. Grafetstätter, Andrea: Ludus compleatur. Theatralisierungsstrategien epischer Stoffe im spätmittelalterlichen und frühneuzeitlichen Spiel. Wiesbaden 2013, S. 133u. S. 141, nimmt - vor allem wegen der Nähe des Großen Neidhartspiels zum Geistlichen Spiel - eine Simultanbühne an, auch wenn dies nicht zu belegen ist. Eine freie, durch Schranken abgetrennte Raumbühne (in oder vor der Stadt) ist ebenso möglich.
} 
mit Stangen bereitstanden, um Verletzte zu bergen. ${ }^{61}$ Wenn die Darsteller nun kurz vor dem eingeschrankten Spielraum ankommen, sollen sie stehenbleiben und den Präcursor alleine in diesen treten lassen. Insofern die Prozession der Darsteller eine Art Präludium darstellt, nimmt der Ausschreier nun die Funktion eines Mittlers zwischen diesem Vorspiel und dem eigentlichen Spiel ein und darf als erster, alleine, den Spielraum besetzen und mit seiner Stimme das Spiel ankündigen (Hört zu Jer Frawenn, V. 24).

Nachdem ein zweiter Präcursor eingetreten ist und die Figuren angekündigt hat, ist im Regiebuch folgendes zu lesen: Darnach geet das Spyl in der / Ordnung in die Schrannckn, Da sullen Stüel vnd pennck / gericht seyn [...] (V. 41-44). Die Darsteller treten jetzt alle in der Prozessionsreihung in den Spielraum und nehmen ihre Plätze, teils auf Bänken und Stühlen, ein. Es wird nun genau angegeben, wo innerhalb des umschrankten Raumes die einzelnen Spielgruppen Aufstellung nehmen sollen:

So sezet sich die Herzogin mit / Jrem zymmer zeöbrist, / Vnd auf ainer Seytn gerravm / herdan, der Neydthardt mit / seiner Ritterschaft vnd Dyenern, / Auf dem anndern Ort gegn über doch üntrer, Ennglmayr vnd seine Gselln [...] (V. 45-52).

Herzogshof, Ritterschaft und Bauern positionieren sich an jeweils anderen Orten innerhalb des Raumes und bilden ein räumliches Dreiecksverhältnis zueinander. Dabei scheinen sich die Bauern in symbolischer Weise unterhalb der Adelsgruppen zu postieren (üntrer, vielleicht auch auf einem Spielfeld mit Gefälle). Alle Darsteller können nun den gesamten Raum zur Aktion benutzen und von überall sichtbar sein. Der umschrankte Raum wird zu einer großen, planen Bühne, welche die Akteure proxemisch und stimmlich vollständig ausnutzen können. Dies wird noch deutlicher, wenn Neidhart sich von der Herzogin verabschiedet hat und nun das Veilchen sucht. Er geht mit seinen Rittern in der Schrannkn / ze rinng vmbe, den veyel ze=/ suchen, Vnd fynndt zelest / myttn im Plaan ainen Veyel (V. 88-93). Die zentrale Szene des Spiels ist also in der Mitte der Spielfläche lokalisiert, es kommt zu einer zentrifugalen Bewegung von den Schranken hin zum Veilchen. Das ze rinng vmbegen, man darf von einem tanzartigen, prozessionalen Schreiten ausgehen, wiederholt sich, wenn Neidhart der Herzogin vom Veilchenfund berichtet und der ganze Hof mit musikalischer Begleitung auf der Innenseite der Schranken umherzieht, oder besser -tanzt, bis man schließlich beim vermeintlichen Veilchen angekommen ist:

\section{[...] vnd geet das}

ganntze Spyl in der Ord=

nung in der Schrannckn

ze rynng $v m b$,

vnd stellent

sich zelest an,

So steent die Pfeyffer auf $\mathrm{Jr}$

Ort, vnd pfeyffent zu Tanntz,

61 Vgl. Ranft, Andreas: »Turnierbücher«. In: Residenzen-Kommission der Akadademie der Wissenschaften zu Göttingen (Hg.): Handbuch Höfe und Residenzen im spätmittelalterlichen Reich. Bd. 15. Ostfildern 2005, S. 635-644, hier S. 640. 
So tanntzt der Neydthardt

mit der Herzogin, auch die

Ritter vid Jungkfrawen,

Vnd die Pawrn mit Jren

Weybn vnd Dyern nach Jn (V. 194-205).

Der Tanz ist das vereinigende Moment der drei Stände Adel, Rittertum und Bauern, und im Moment des musikalischen Einsatzes dürfen alle Spieler an den Schranken entlang im Kreis tanzen. Sobald die Musik zu Ende ist, nehmen die Spielgruppen wieder ihre Plätze ein - ein proxemisches Prinzip, das der Simultanbühne mit ihren Häusern und Ständen stark ähnelt und von ihr übernommen scheint. Wichtig ist aber hier, dass die Spielgruppen mit ihren vektoralen Bewegungen, ihrer Motorik und ihren Choreographien den Raum wechselseitig besetzen und wieder freimachen, sie >erspielen< sich quasi den theatralen Raum im Durchgang durch die verschiedenen Szenen des Spiels. Das Handeln erfolgt sukzessiv nach der Szenenfolge des Veilchenschwanks, aber auch simultan in den intermittierenden Momenten des gemeinsamen Tanzens und Prügelns. Statt einer polaren theologischen Logik haben wir eine sozial markierte Ständeeinteilung (die sich räumlich in der wechselnden Dreiecksbeziehung der Spielgruppen manifestiert), die andererseits aber auch über starke parodistische und karnevaleske Züge des Spiels karikiert und verlacht werden kann. ${ }^{62}$

Nach der Entehrung und Verbannung Neidharts kommt es nochmals zu einer zentralen Szene (V. 271: tritt Neydthardt [...] mytten in Plaan), der Klage des Ritters über sein Schicksal, worauf sich nach V. 315 beginnend mit Engelmars Auftritt das Spiel zu einer Art Reihenspiel wandelt, bei dem jeweils ein Ritter und ein Bauer zu Neidhart kommen und ihre Redeeinsätze haben. Die Bühne füllt sich allmählich, bis schließlich noch zwei Fässer hereingezogen werden (Faßschwank), um die Rache an den Bauern noch etwas zu verstärken. Es ließe sich von einem crescendo der Zahl der Darsteller, aber auch des Rufens und Schreiens auf der Bühne sprechen. Hier wird selbstverständlich keine Heilserfahrung gemacht, sondern das Publikum soll und darf über die Geschehnisse und die Figuren herzhaft lachen. Die Schranken zeigen sich schließlich als ein umschließender Raum, in welchem sich ansatzweise ein fiktionales Geschehen abspielt, an dem auch die Zuschauer körperlich durch die Nähe zum Spielplan und den Darstellern sowie durch die Vorgänge auf dem Spielfeld angesteckt werden. Dies ist ein Kennzeichen des populären Theaters: Neben dem grobdrastischen Sprechen und Handeln der Bauern, neben dem Zusammenbinden von höfischem Zeremoniell und menschlichen Ausscheidungen, den disproportioniert scheinenden Klagen von Herzogin und Ritterschaft und dem omnipräsenten Tanz, ist es vor allem der umschrankte, frei verfügbare und in seinen Grenzen vollständig ausspielbare Spielraum, welcher ein spezifisches Näheverhältnis zum Publikum herstellen kann, das der Bühnensituation der Einkehrspiele ähnelt, jener des geistlichen Spiels jedoch diametral entgegen gesetzt ist - nimmt

62 Vgl. zum Verhältnis von Raum, Parodie und Gelächter Velten, Hans Rudolf: Scurrilitas. Das Lachen, die Komik und der Körper in Literatur und Kultur des Spätmittelalters und der Frühen Neuzeit. Tübingen 2017, S. 240-252. 
man die erwähnten festen Plätze der Spielgruppen auf dem Spielplan, in die sie sich zurückziehen können, einmal aus.

\subsection{Aufführungen von Rosengärten}

Ich werde nur kurz und kursorisch auf eine zweite Form des weltlichen Spiels eingehen, das ebenfalls mit Schranken aufgeführt wurde: die Dramatisierung des heldenepischen Stoffes von Kriemhilds Rosengarten zu Worms. Aufführungen von Rosengärten sind in Wesel, Langensalza und Windsheim belegt, an Texten haben wir zwei Tiroler Spiele, die in ca. 30 Textzeugen überliefert sind, und ein Berliner Fragment. ${ }^{63} \mathrm{Ob}$ es sich dabei eher um Schauspiele oder um turnierartige Wettkämpfe handelt, ist schwer zu entscheiden. ${ }^{64}$

Der Rosengarten von Wesel ist aufführungshistorisch am besten belegt; er wurde zwischen 1380 und 1428 siebenmal veranstaltet und schlug sich daher in den Stadtrechnungen nieder. Es wurde von den Weseler Schneidern aufgeführt, weshalb der Rechnungsschreiber den Rosengarten auch ludus sartorum nennt. »Die Schneider >spielten< ihren Rosengarten auf dem Markt, der mit Schranken umzäunt (Pfostenlöcher) und mit Sand bestreut wurde. $\aleph^{65}$ Hier wird der Zusammenhang mit den Schauturnieren des städtischen Adels deutlich, vielleicht auch mit Spottturnieren, die im 15. Jahrhundert als Gesellschaftsspiele und Attraktionen für das gesamte städtische Publikum immer beliebter wurden. ${ }^{66}$ Dazu passt, dass Simon den ludus sartorum nicht als Schauspiel, sondern als Turnier bezeichnet, worin der Zusammenhang zur Praxis spätmittelalterlicher städtischer Ritterspiele deutlich wird. ${ }^{67}$ Allerdings kann ein Turnier von Schneidern kaum die Repräsentationsform adliger Turniere erreichen; es muss im Gegenteil als zugehörig zur »verkehrten Welt « karnevalesker performances gesehen werden und damit würde sein Unterhaltungswert im Zentrum stehen. ${ }^{68}$

Das Turnierspiel der Schneider beruhte auf dem beliebten Dietrichepos vom Rosengarten zu Worms. Analog zum höfischen Ritterspieltypus >Sturm und Eroberung des Hauses< wird es den Kampf um den von Kriemhild gepflegten Rosengarten imitiert haben, den Dietrich von Bern und seine Recken angreifen und den König Giebichs Riesen, unter Führung des hürnen Seyfried, verteidigen. ${ }^{69}$ Allein die

\footnotetext{
63 Vgl. Simon (s. Anm. 26), S. 26-30, sowie zu den Tiroler Spieltexten ausführlich Grafetstätter (s. Anm. 60), S. 236-270.

64 Vgl. ebd., S. 236.

65 Simon (s. Anm. 26), S. 26.

66 Vgl. dazu Retemeyer, Kerstin: Vom Turnier zur Parodie. Spätmittelalterliche Ritterspiele in Sachsen als theatrale Ereignisse. Berlin 1995, S. 44-46; 62-66. Zur Funktion von Schranken bei Turnierspielen vgl. ebd., S. 127-129.

67 Simon vermutet das, weil in der Rechnung von 1395 steht: doe dye scrodere den rosengarde vuchten (ders. [s. Anm. 26], S. 26). Da vechten auch >sich prügeln< bedeutete, wird die Ambivalenz der Handlungen deutlich und damit eine Turnierparodie wahrscheinlicher.

68 Vgl. die Analysen von Turnieren als Possenspielen (Scherzturniere der Handwerker, Gesellenstechen, Kübelstechen, Bauernstechen) bei Retemeyer (s. Anm. 66), S. 98-184.

69 So die Vermutung Simons (s. Anm. 26), S. 26f. Vgl. zum Ritterspieltypus Retemeyer (s. Anm. 66), S. 44-76; darunter finden sich auch sogenannte Minneburgspiele, etwa in Zittau und Dresden.
} 
Tatsache, dass Schneidergesellen die heldenepischen Recken und Riesen gespielt haben, lässt an eine parodistische Anlage des Spiels denken. Andrea Grafetstätter hat in ihrer Untersuchung zur Theatralisierung heldenepischer Stoffe nicht nur das Weseler Spiel, sondern Rosengartenspiele überhaupt in dieser Perspektive untersucht: »Das Stadtpatriziat [...] versucht, sich durch die Imitation überkommener feudaler Inszenierungen als neue Elite zu legitimieren. « ${ }^{70}$ Grafetstätter bezeichnet die Theatralisierungen heldenepischer Stoffe als >Unterhaltungstheater $<$ und arbeitet ihre vielfältigen komischen Aspekte (Verspottung des überkommenen ritterlichen Tugend- und Minnekodex, Karikatur ritterlicher Turnierpraktiken) heraus. Es ist anzunehmen, dass die Schranken hier nicht allein den Spielplatz eingrenzen, sondern zur prozessionalen Schau und Repräsentation dienten. So ist von einem grotesken Bauernstechen in Dresden überliefert, dass die als Ritter gekleideten Bauern »auf Seiner Fürstlichen Gnaden Befehl dreimal in der Ordnung um die Schranken reiten und sich sehen lassen müssen. «11 Interessant ist auch, dass die emotionale Beteiligung der Zuschauer an solchen turnierartigen Veranstaltungen offensichtlich sehr hoch war, sodass die Errichtung von Schranken zur Sicherheit aller Beteiligten teils mit sehr hohem Aufwand verbunden war. ${ }^{72}$

Das Windsheimer Spiel mit dem Rosengarten (Neumann 3338) weist noch eine weitere Verwendung von Schranken auf, die sich an die Tradition der Tafelrunden (table ronde, tavola rotunda) anlehnten. Das waren literarische, am Artusstoff und seinem Inventar orientierte höfische Turniere, in deren Mittelpunkt ritterliche Zweikämpfe mit stumpfen Waffen standen. Dafür wurde ein großer Platz mit Schranken abgesteckt, an dessen Rand die Zuschauer und Kampfrichter Platz nahmen. ${ }^{73}$ Das Windsheimer Spiel weist nun innerhalb des Spiel- oder Kampfplatzes ein Dekorationselement auf, welches analog zum Zelt der Königin der Tafelrunde in der Mitte des Platzes stand: Es war offensichtlich eine umzäunte Gartenkulisse, in dem sich Kriemhild befand (der Rosengarten als Schauobjekt). ${ }^{74}$ Es ist zu vermuten, dass die Kämpfe zwischen Dietrichs Recken und Kriemhilds Riesen sich an den Grenzen dieses Gartenraumes abspielten und dass die Absperrung die beiden Kampfgruppen voneinander trennte. Einen Hinweis darauf geben die Regieanweisungen im späteren Tiroler Reckenspiel, dem vasnacht spill von den risn oder reckhnn, das Vigil Raber 1511 in Bozen aufgezeichnet hat und in dem Anweisungen wie Asprian springt in den Garten / vnd spricht (Z. $261 \mathrm{f}$.) zu finden sind.$^{75}$ Das Einkehrspiel kann einen solchen Sprung nur in verhaltener Form zur Aufführung bringen, doch auf einem Spielplan im Freien ist das Überspringen einer Abgrenzung oder eines Zaunes nicht nur möglich, sondern kann spektakulär inszeniert werden. Es kommt dabei auch zu einer Reziprozität der Schranken im Spiel und als Abgrenzung vom Spiel, was

\footnotetext{
70 Grafetstätter (s. Anm. 60), S. 236.

71 Zitat bei Retemeyer (s. Anm. 66), S. 142f.: »Der Ritt um die Schranken beschrieb das Turnierfeld, den eingegrenzten Raum, in dem gekämpft werden sollte $\ll$.

72 Vgl. ebd., S. 62 mit Hinweis auf das 1389 von Herzog Johann von Görlitz veranstaltete Turnier.

73 Vgl. dazu die Beschreibung bei Bumke, Joachim: Höfische Kultur. Literatur und Gesellschaft im hohen Mittelalter. 2 Bde. München 1986, Bd. 1, S. 363.

74 Vgl. Simon (s. Anm. 26), S. 28.

75 Vgl. ebd.
} 
den Aspekt der Porosität solcher Grenzen und die Möglichkeit ihrer Überwindung wiederum deutlich macht.

Fasst man diese Angaben zu den Rosengartenspielen zusammen, muss ein medien- und gattungsspezifischer Zusammenhang von Schranken und populärem Spiel bestanden haben, welches zur Unterhaltung und zur Freude eines überständischen Publikums dienen sollte. Anlässe für das Lachen des Publikums waren parodistische und satirische Bezüge, aber auch Bewegungsintensität, groteske Körperlichkeit und gespielte Gewalt. Die vorherrschende Rezeptionshaltung ist somit von Ausgelassenheit, Dispersion und aktiver Teilhabe geprägt. Dies steht ganz im Gegensatz zur Simultanbühne geistlicher Spiele, welche ja die Aufmerksamkeit des Publikums bündeln sollte, um durch Immersion ins Spiel $^{76}$ und meditative und empathische Betrachtung des Leidens Heilserfahrung zu machen und Ablass zu erlangen.

\section{Funktionale Raumanalyse des umschrankten Spielraums}

Wenn die Konstitution des mittelalterlichen Theaters an die Geschichte von Bühnenformen und den von ihnen geschaffenen Räumen und Raumqualitäten als Rahmenkonzepte gebunden ist, wenn theatraler Raum sich durch »Koproduktionen von Akteuren und Zuschauern an einem Ort ${ }^{77}$ konstituiert, dann kann die Simultanbühne nicht als paradigmatisches Aufführungsprinzip geltend gemacht werden. Für eine Aufführungsgeschichte des mittelalterlichen Theaters müssen alle überlieferten Bühnenformen von 1300 bis 1600 in Betracht gezogen werden. Eine dieser Bühnenformen ist der umschrankte Spielraum, wie er sich in zahlreichen weltlichen, aber auch in einigen geistlichen Schauspielen manifestiert. Schranken strukturieren den offenen, theatralen Raum zirkulär und prozessional, sie unterscheiden sich als performatives Dispositiv deutlich von anderen theatralen Raumprinzipien und haben eine andere Wirkung auf die Zuschauer.

Mittelalterliches Schauspiel muss zunächst einmal, unabhängig von der Aufführungsform, als ein theatrales Handeln begriffen werden, das »durch seine Bewegung sich so etwas wie Bühne erst erspielt $«{ }^{78}$ Keine Bühnenform des Mittelalters ist in Bezug auf das Verhältnis von Darstellern und Zuschauern so normativ und richtunggebend bzw. sogar einschränkend wie die neuzeitliche Guckkastenbühne. Die Differenzierung in aktiven Vollzug und passives Zuschauen (die im modernen Theater wieder rückgängig gemacht wird), die Aufteilung in Zeigen und Wahrnehmen ist dem mittelalterlichen Spiel nicht eigen. Vielmehr trägt es rituelle Merkmale der Prozession, des Ein- und Auszuges, des Umgehens und ist daher vor allem durch ständige Bewegung und Fluidität charakterisiert, die von einer $>$ Bühne $<$ gar nicht repräsentiert werden können. Das Konzept (und das Wort) >Bühne $<$ ist ohnehin erst ein Resultat der Neuzeit und im Mittelalter kein verwendeter Begriff. Selbst auf engen Spielflächen wird seit dem frühen Mittelalter oft im Kreis gegangen oder auf der Stelle getreten. Durch das Abschreiten von Wegen werden sowohl in Innen-

\footnotetext{
76 Vgl. dazu Ackermann (s. Anm. 55).

77 Roselt (s. Anm. 4), S. 267.

78 Kirchner (s. Anm. 18), S. 9.
} 
räumen (Kirchen, Rathäusern) und in Außenräumen (Simultanbühnen, umschrankte Spielflächen) Räume geschaffen, indem sie körperlich und haptisch erschlossen und besetzt werden. ${ }^{79}$ Die Bilder und figurae, die Kämpfe und Konflikte, die in diesen Räumen gezeigt werden, sind ursächlich mit dem topographischen Raum der Aufführung verbunden.

Während die Simultanbühne darauf ausgelegt ist, das Heilsgeschehen des geistlichen Spiels den Zuschauern zu vergegenwärtigen, ist die Funktionalität und Medialität des mit Schranken begrenzten offenen Spielraums anders gelagert. Ich möchte abschließend einige Punkte zusammenfassen, um dieses mittelalterliche Aufführungsdispositiv zumindest ansatzweise zu charakterisieren:

1. Schranken sind sehr einfache, ephemere und mobile Hilfsmittel der räumlichen Organisation von Darsteller- und Zuschauerraum. Sie sind raumschaffend, indem sie aus einem Spielfeld einen Raum machen. Sie können jederzeit auf- und abgebaut werden, die Spielfläche vergrößern oder verkleinern und stellen somit eine Nullstufe der Bühne dar.

2. Sie ordnen die Räume von Darstellern und Zuschauern auf derselben Höhe an und organisieren ihr Verhältnis in Form eines kreis- oder halbkreisförmigen theatralen Raums (rynng, circulus), wobei Darsteller und Zuschauer meist stehen, aber auch die Möglichkeit erhalten können, sich zu setzen.

3. Durch diese eher fluide Form des vertikalen und horizontalen Verhältnisses, welche auf der Seite der Zuschauer durch Enge charakterisiert ist, ergibt sich eine gewisse Porosität der Grenze zwischen Darstellern und Zuschauern. Diese Durchlässigkeit ist mit einer intensiven Kommunikation und wechselseitiger Entgrenzung wie beim Straßentheater verbunden, sodass hier nur ein schwacher Eindruck von Institutionalität und Herrschaft entsteht (anders als bei einer erhöhten, zentralen Podiumsbühne).

4. Schranken dienen nicht allein der Abgrenzung des Spielfeldes, sondern auch der Schaffung seiner Zu- und Abgänge (Ein- und Auszüge der Darsteller), was eine andere, wesentlich von Nähe bestimmte visuelle Wahrnehmung ermöglicht. Einund Auszüge haben durch ihren Repräsentationscharakter einen besonders hohen Schauwert, bei dem die Nähe ein maßgeblicher Faktor ist.

5. Durch die bewusste Positionierung der Darsteller und Spielgruppen im umschrankten Raum kann dieser proxemisch und symbolisch erzeugt und strukturiert werden (wie im Neidhartszenar zu erkennen ist).

6. Schranken als Medien der Ein- und Abgrenzung bieten vielerlei Möglichkeiten für ihre anderweitige Nutzung: für die prozessionale Bewegung in den umschrankten Bereich hinein und entlang der Schranken, für das Umspielen der Schranken innerhalb des Spielplans, für die Überwindung der Schranken, für den Tanz innerhalb und außerhalb der Schranken (Neidhartspiel).

7. Falls Schranken innerhalb des Publikums aufgebaut werden, schaffen sie räumlich abgegrenzte Abschnitte für gesellschaftliche Rangunterschiede, welche auch symbolisch sichtbar sind.

\footnotetext{
79 Vgl. ebd., S. 62 u. S. 90: »Das Besetzen eingegrenzter Räume ist ein gestaltendes Element zunächst des liturgischen Vollzugs «.
} 
8. Schranken und offener Spielplan bieten für viele Zuschauer, die nicht vorne stehen, nur eine eingeschränkte Sicht und eingeschränkte Akustik, da die Entfernung von den Darstellern je nach Standort unterschiedlich ist. Dies führt zu einer weniger auf Semantik und Didaktik ausgerichteten Rezeption; stattdessen ist sie wesentlich abhängiger von lauten Ereignissen und den Reaktionen der anderen Zuschauer.

9. Schranken werden als Aufführungsdispositiv vor allem in weltlichen, oft fastnächtlichen Spielen verwendet, die einen überständischen, popularen Charakter besitzen, vom gemeinschaftlichen Lachen regiert werden und der Unterhaltung im Zeichen der semantischen Inversion dienen. Schranken sind dem gemeinsamen Lachen und der Unterhaltung insofern förderlich, als sie das Publikum in eine bestimmte räumliche Nähe zwingen. Dies hat die Schrankenbühne mit der schrankenlosen Wirtshausbühne gemeinsam.

10. Schranken als Aufführungsdispositive stellen einen Zusammenhang her zwischen unterhaltenden Schauspielen (etwa literarische Theatralisierungen wie den Rosengartenspielen) und unterhaltenden Ritualen wie Turnierspielen.

11. Aufführungen auf umschrankten Spielflächen erlauben die Erkenntnis theatraler >als-ob<- Handlungen und erlauben es dem Spiel, durch Komik Distanz vom gezeigten Geschehen zu schaffen.

12. Der umschrankte Spielraum entzieht sich der normativen Logik von Simultaneität und Sukzessivität, indem er beides ermöglicht (wie am Alsfelder Passionsspiel und am Neidhartszenar zu erkennen).

Funding Open Access funding enabled and organized by Projekt DEAL.

Open Access Dieser Artikel wird unter der Creative Commons Namensnennung 4.0 International Lizenz veröffentlicht, welche die Nutzung, Vervielfältigung, Bearbeitung, Verbreitung und Wiedergabe in jeglichem Medium und Format erlaubt, sofern Sie den/die ursprünglichen Autor(en) und die Quelle ordnungsgemäß nennen, einen Link zur Creative Commons Lizenz beifügen und angeben, ob Änderungen vorgenommen wurden.

Die in diesem Artikel enthaltenen Bilder und sonstiges Drittmaterial unterliegen ebenfalls der genannten Creative Commons Lizenz, sofern sich aus der Abbildungslegende nichts anderes ergibt. Sofern das betreffende Material nicht unter der genannten Creative Commons Lizenz steht und die betreffende Handlung nicht nach gesetzlichen Vorschriften erlaubt ist, ist für die oben aufgeführten Weiterverwendungen des Materials die Einwilligung des jeweiligen Rechteinhabers einzuholen.

Weitere Details zur Lizenz entnehmen Sie bitte der Lizenzinformation auf http://creativecommons.org/ licenses/by/4.0/deed.de. 